\title{
r-Process Contributions to Low-Metallicity Stars
}

\author{
Friedrich-K. Thielemann ${ }^{1,2, *}$, Khalil Farouqi ${ }^{3}$, Stephan Rosswog ${ }^{4}$, and Karl-Ludwig Kratz ${ }^{5,6}$ \\ ${ }^{1}$ Dept. of Physics, University of Basel, Klingelbergstrasse 82,CH-4056 Basel, Switzerland \\ ${ }^{2}$ GSI Helmholtz Center for Heavy Ion Research, Planckstrasse 1, D-64291 Darmstadt, Germany \\ ${ }^{3}$ ZAH, Landessternwarte, University of Heidelberg, Königstuhl 12, D69117 Heidelberg, Germany \\ ${ }^{4}$ The Oskar Klein Centre, Department of Astronomy, Stockholm University, Stockholm, Sweden \\ ${ }^{5}$ Dept. of Chemistry, Pharmacy \& Geosciences, University of Mainz, D-55126 Mainz, Germany \\ ${ }^{6}$ Max Planck Institute for Chemistry (Otto Hahn Institute), D-55128 Mainz, Germany
}

\begin{abstract}
Various nucleosynthesis studies have pointed out that the rapid neutron capture r-process elements in very metal-poor (VMP) halo stars might have different origins. It has been known that an r-process can either be obtained in neutron-rich low $Y_{e}$ conditions or in high entropy environments [see e.g. 1-5], an overview over many investigations has appeared recently [6]. In the present article we analyze with statistical methods the observational abundance patterns from trans-Fe elements up to the actinides and come to the conclusion that four to five categories of astrophysical events must have contributed. These include the ejection of $\mathrm{Fe}$ and trans-Fe elements $\mathrm{Sr}, \mathrm{Y}, \mathrm{Zr}$ (continuing possibly beyond to slightly higher mass numbers) in category 0 events (hereafter " $\mathrm{C} 0$ "), $\mathrm{Fe}$ and weak r-process contributions (including Eu in moderate to slightly larger but varying amounts) in CI and CII events, strong r-process abundance patterns with no or negligible (in comparison to solar) Fe production in CIIIa and CIIIb events, where category CIIIb shows a tendency for an actinide boost behavior. When comparing these categories with presently existing nucleosynthesis predictions, we suggest to identify them (despite remaining uncertainties) with regular core-collapse supernovae, quark deconfinement supernovae, magnetorotational supernovae, neutron star mergers, and outflows from black hole accretion tori.
\end{abstract}

\section{Introduction}

Stellar (surface) abundances are inherited from the interstellar medium in which the stars were born, i.e. one can look back in time. The early phases of galactic evolution are characterized by a large degree of abundance scatter and spatial inhomogeneities, which cannot be explained by traditional chemical evolution studies [7]. To account for this, inhomogeneous "chemical evolution" models do not assume that ejecta mix completely and instantaneously with the whole galactic interstellar medium. Instead, they consider only the pollution of about $5 \times 10^{4} \mathrm{M}_{\odot}$ (via a Sedov-Taylor blast wave) of the interstellar medium [see e.g. 8, 9]. This is based on an analysis that the ejection of $0.1 \mathrm{M}_{\odot}$ of Fe leads in a CCSN remnant to $[\mathrm{Fe} / \mathrm{H}] \approx-$ 2.7 [10]. If only $10 \%$ or $1 \%$ of supernova ejecta contribute to the formation of the next star, one expects, therefore, $[\mathrm{Fe} / \mathrm{H}]$ values as small as -4 or -5 . More extended recent chemical

\footnotetext{
*e-mail: f-k.thielemann@unibas.ch
} 
evolution studies take this into account in a more general approach [see e.g. 11, 12]. We will use as a starting hypothesis that stars with metallicities below -2.5 might be interpreted as the result from the contribution of only a single nucleosynthesis event. Can we therefore see in such low-metallicity stars the results of individual but different astrophysical events and which of these events are responsible for the heavy element r-process production?

One of the major questions about r-process contributions to solar abundances is whether they are universal and stem from a unique astrophysical site. Early signs from low-metallicity stars seemed to show such a unique r-process pattern, therefore pointing to a universal site [13]. This interpretation started to become questionable when actinide-boost stars were observed [14] and the first limited-r stars were discovered [15]. As an extension of explaining these different patterns we suggest, despite substantial uncertainties, an identification of the observational categories listed in the abstract with regular core-collapse supernovae (C0), quark deconfinement supernovae (CI), magneto-rotational supernovae (CII), neutron star mergers with a long-lived central remnant (CIIIa) and black hole-accretion torus systems (CIIIb) formed either by collapsars, neutron star black hole binaries or massive neutron star mergers that rapidly form a central black hole. But before doing so and checking these suggestions via utilizing ejecta composition predictions for comparisons with abundance patterns of low-metallicity stars, we want to briefly summarize the statistical methods which led to these identifications.

\section{Statistical Analysis of r-Process Element Abundances in Low-Metallicity Stars}
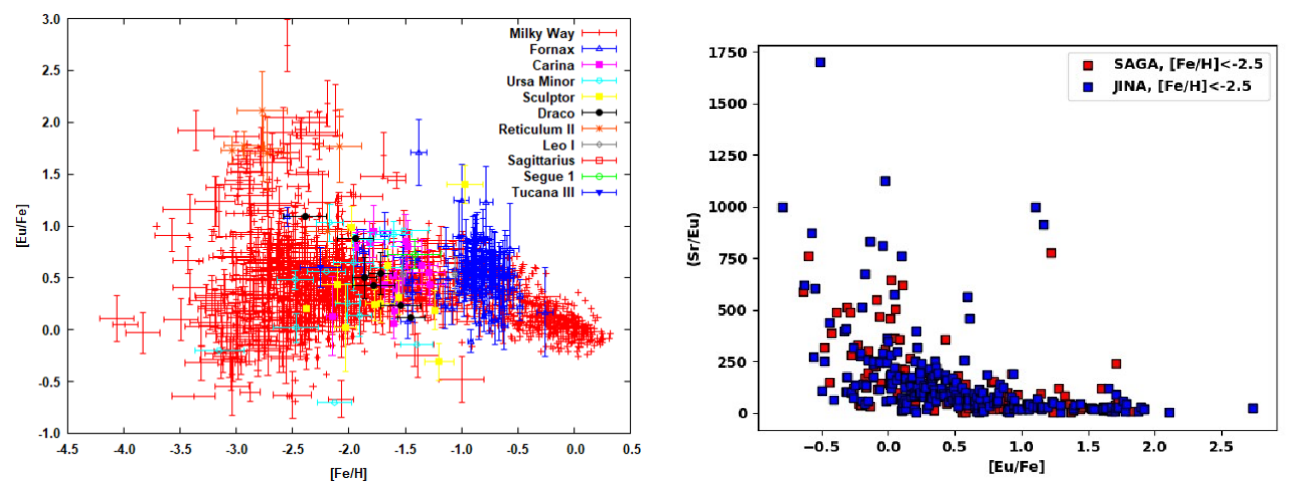

Figure 1. Left: $[\mathrm{Eu} / \mathrm{Fe}]$ ratios of 1572 stars with Eu detections from the SAGA database [16]. A hugh scatter can be observed before at about $[\mathrm{Fe} / \mathrm{H}]=-2$ an averaging (smaller spread) sets in, which continues with a different gradient when SNe Ia start to contribute at -1 . Right: $\mathrm{Sr} / \mathrm{Eu}$ ratios for all stars with $[\mathrm{Fe} / \mathrm{H}<-2.5$, which show a drastic change at about $[\mathrm{Eu} / \mathrm{Fe}]=0-0.3$, i.e. the division between limited-r stars and r-enriched stars [for these observational classifications and the definition of actinide boost stars see e.g. 17-26]. (Notice that some points are identical for SAGA and JINA and only the entry plotted for JINA - in blue - is visible, especially the value of about 1100 for $[\mathrm{Eu} / \mathrm{Fe}] \approx 0$.)

If abundance patterns of low-metallicity stars result from the pollution by only a single type of event, this would cause correlations between those elements resulting from the originating event [for a recent example see 27]. Such correlations (i.e. linear relations, respective constant ratios, between their abundances) underline the co-production of these elements. If (at low metallicities) elemental abundances are interpreted as imprints of individual explosions, 
a large scatter as a function of $[\mathrm{Fe} / \mathrm{H}]$ points to either rare events with different amounts of admixtures and/or to different types of events with strong variations in their ejected composition. The variation in $[\mathrm{Eu} / \mathrm{Fe}]$ vs. $[\mathrm{Fe} / \mathrm{H}]$ could be interpreted by such explanations, the large $\mathrm{Sr} / \mathrm{Eu}$ in limited-r stars vs. the low values in r-enriched stars hints at different types of events (see Fig.1). There exist of course uncertainties in existing data, especially if an easier to detect element like $\mathrm{Sr}$ is compared to a harder to detect element like $\mathrm{Eu}$, that might be close to detection limits. One of the possible options to verify the size of these uncertainties is to compare two databases which contain collected but differently processed information. While the SAGA database is known to enter data from different authors and observational facilities without much censorship, the JINAbase library has been compiled, addressing also systematic errors. When utilizing the SAGA or JINA databases for Fig,1 (right panel) we get slightly different but very similar results with the same trend. Therefore, we think that the conclusions pointing to different origins, due to this trend, are sound.

In late phases of galactic evolution, a large number of different events have taken place, leading, due to superpositions and admixtures of the ejecta of many different types of events, also to close to constant element ratios. These represent, opposite to the behavior at lowest metallicities, "spurious" correlations. However, they are just the result of averaging over many events and do not support the interpretation as co-production in an individual event. In order to analyze the observed abundance patterns in low-metallicity stars in a safe way among the above listed interpretations, we utilize mainly three methods to gain insight into the possibly different origins of the r-process elements: cluster analysis, rank tests, and correlations.

\subsection{Clustering}

Cluster analysis is a statistical technique to identify how data can be grouped because of characteristics they have in common [28]. It sorts data into groups which have a maximal number of characteristics in common. One method is the K-means Clustering: it distributes data into K mutually exclusive clusters so that with a given measure data points are as close to each other as possible within each cluster, while at the same time they as far from other clusters as possible. Each cluster is characterized by a central point.
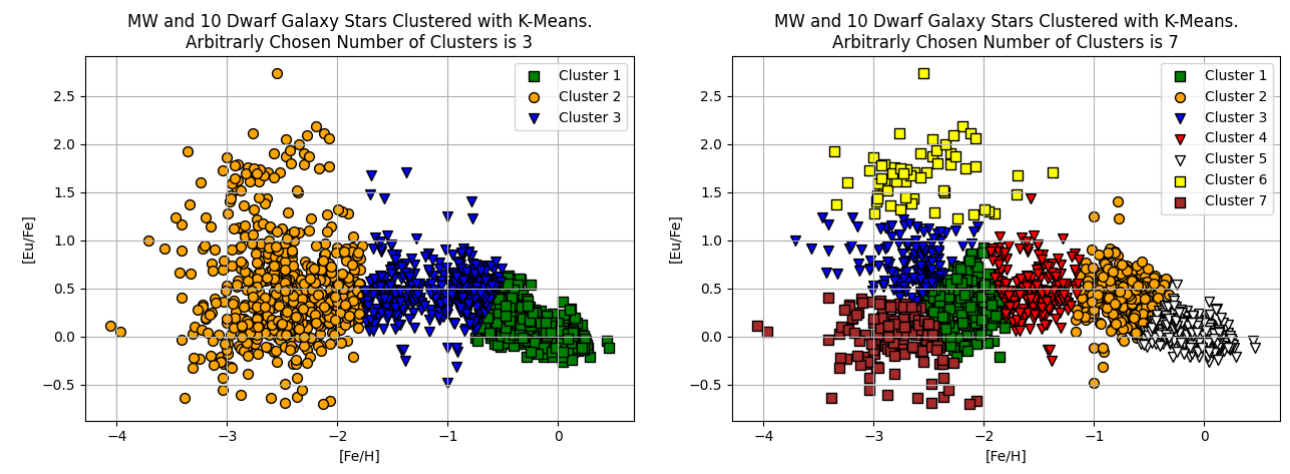

Figure 2. 3-cluster and 7-cluster analysis of $[\mathrm{Eu} / \mathrm{Fe}]$ ratios as a function of $[\mathrm{Fe} / \mathrm{H}]$, see text.

In the 3-cluster analysis of the Eu vs. Fe abundances of Fig.2 one can recognize in cluster 1 the low-metallicity stars where the large scatter indicates contributions from individual rare and/or different events, in cluster 2 for $[\mathrm{Fe} / \mathrm{H}]>-2$ that already an averaging/mixing of event products sets in and the scatter becomes small, and in cluster 3 that $\mathrm{Fe}$ is contributed also from 
$\mathrm{SNe}$ Ia and changes the general $\mathrm{Eu} / \mathrm{Fe}$ trend. In the 7-cluster analysis one recognizes different stages of the approach to averaging/mixing (clusters $1,2,4)$ and for low-metallicity stars with $[\mathrm{Fe} / \mathrm{H}]<-2.5$ three clusters $(3,6,7)$ with properties close to the observational limited-r, r-I, and $\mathrm{r}-\mathrm{II}$ classes of $[\mathrm{Eu} / \mathrm{Fe}]<0-0.3,<1$, and $>1$.

\subsection{Rank Test}
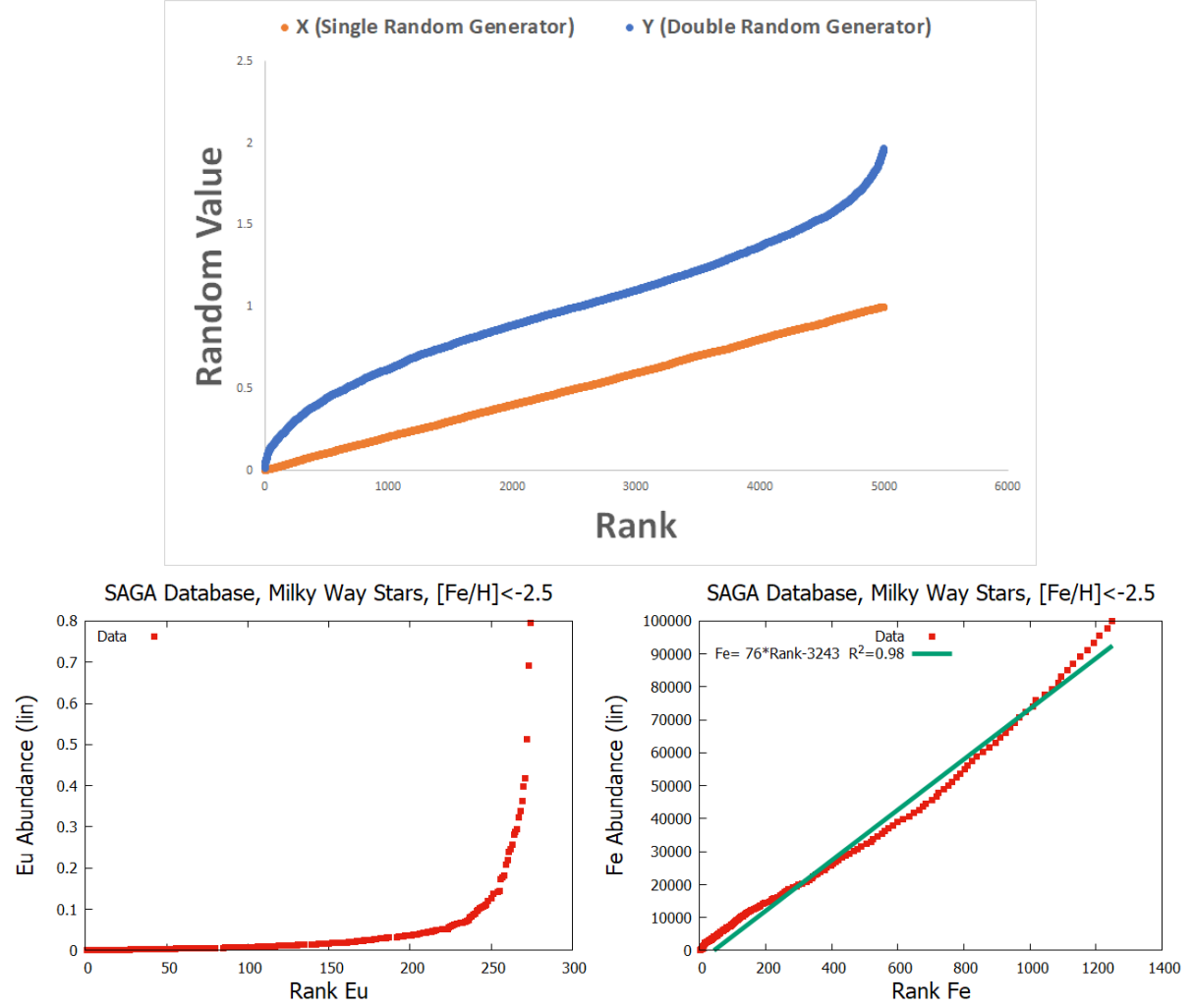

Figure 3. Top: data ordered along their ranks from a single number generator $X$, showing (a) a linear relation between $X$-values and their rank, and (b) the sum $X_{1}+X_{2}$ of two single number generators vs. its rank, deviating from a linear relationship. Bottom left: Eu abundances vs. their rank for low-metallicity stars with $[\mathrm{Fe} / \mathrm{H}]<-2.5$, at high ranks (i.e. high Eu abundances) one recognizes an additional/different strong contribution. Bottom right: The same for Fe abundances, displaying almost a linear relationship, pointing to one identical source, but at low as well as high Fe abundances slight deviations are seen.

We show examples for utilizing a single random number generator $X$ and ordering the values according to their size, giving them integer entries (ranks). When plotting them as a function of their ranks, one can see a linear relation between the random numbers and their ranks (see the orange line in Fig.3, top panel). The blue line is obtained when utilizing two random number generators, adding their values $Y=X_{1}+X_{2}$, and plotting $Y$ as a function of its ranks. It deviates from a linear relation between $Y$ and its ranks. The deviation at low ranks depends on the fact whether the second contribution includes many low-value entries. The deviation at high ranks depends on the number of high-value entries. If one applies this method to 
abundance observations of individual elements as a function of rank, it can indicate whether only one type of nucleosynthesis source or two or several contributed to this element.

We utilized this method also to display Eu abundances versus their corresponding ranks in Fig.3 (bottom left). The integer rank passes, with increasing Eu abundances, through all observational points from the smallest to the highest abundance. The fit deviates strongly from a linear behavior at high Eu ranks. This argues for a superposition with an additional Eu source, especially responsible for the high Eu abundances. A missing deviation from the linear slope at small ranks underlines that this additional Eu source contains essentially no contributions with negligible Eu production in Fig.3 (bottom right). A different behavior can be noticed for Fe abundances versus their corresponding ranks. The figure shows a close to linear relation. This would argue for a single or dominating production site (explosive Si-burning in CCSNe?). The slight deviation at high ranks could be interpreted as an additional source with high Fe production (hypernovae?), the slight deviation at low ranks as an additional source with small Fe production (magneto-rotational supernovae?)
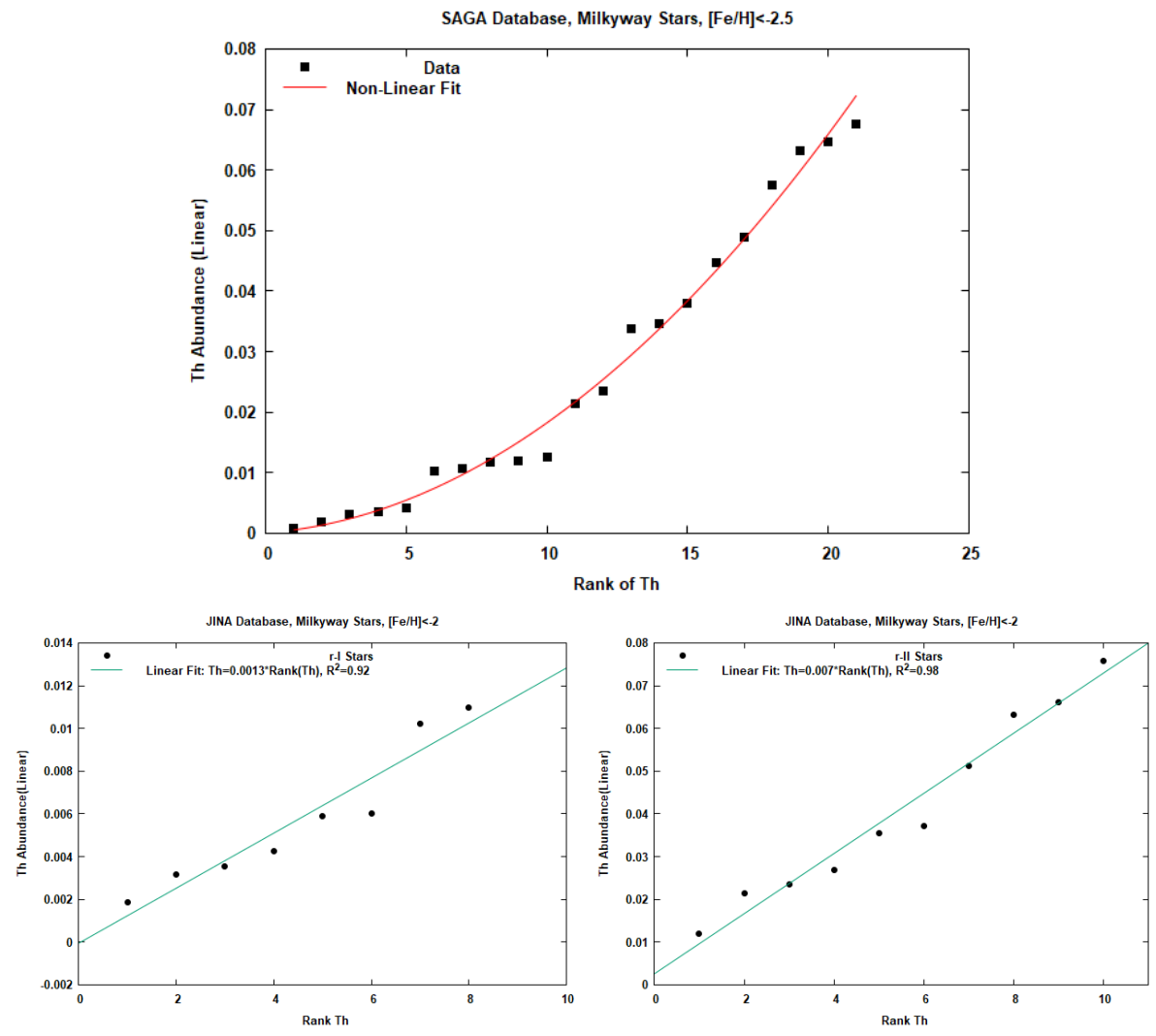

Figure 4. Top: Th abundances vs. their rank for low-metallicity stars, i.e. r-enriched r-I and r-II stars. Bottom left and right: The same but considering r-I and r-II stars separately. The linear relationship points to a single nucleosynthesis source for each of these two sets.

If one performs a similar rank test for Th (Fig,4) in r-enriched stars (r-I and r-II), it can be noticed that a superposition of two types of events is required. But one finds a linear rank relation when plotting Th abundances as a function of their ranks separately for $\mathrm{r}-\mathrm{I}$ 
and r-II stars (with a slight scatter). Thus, r-I and r-II stars seem each to be dominated by contributions from one specific type of event, each of them being responsible for their Th production independently. This is not the case when performing a rank tests for Eu in r-I and r-I stars (Fig.5). Opposite to Th, Eu does not show a linear rank relation, when considering $\mathrm{r}$-I and r-II stars separately. This suggests that also among r-enriched (strong r-process r-I and r-II) stars weak r-process events «spill in» an additional Eu contribution.
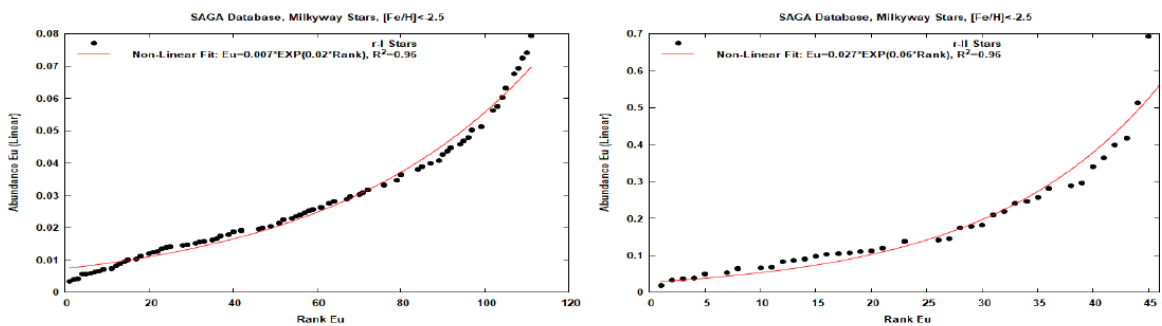

Figure 5. Rank tests for Eu abundances in r-I and r-II stars separately. For Eu a further nucleosynthesis source is required in addition to the two different strong sources dominating Th in r-I and r-II stars. This indicates that $\mathrm{Eu}$ from a weak r-process event (dominating limited-r stars) contributes as well.

\subsection{Correlations}

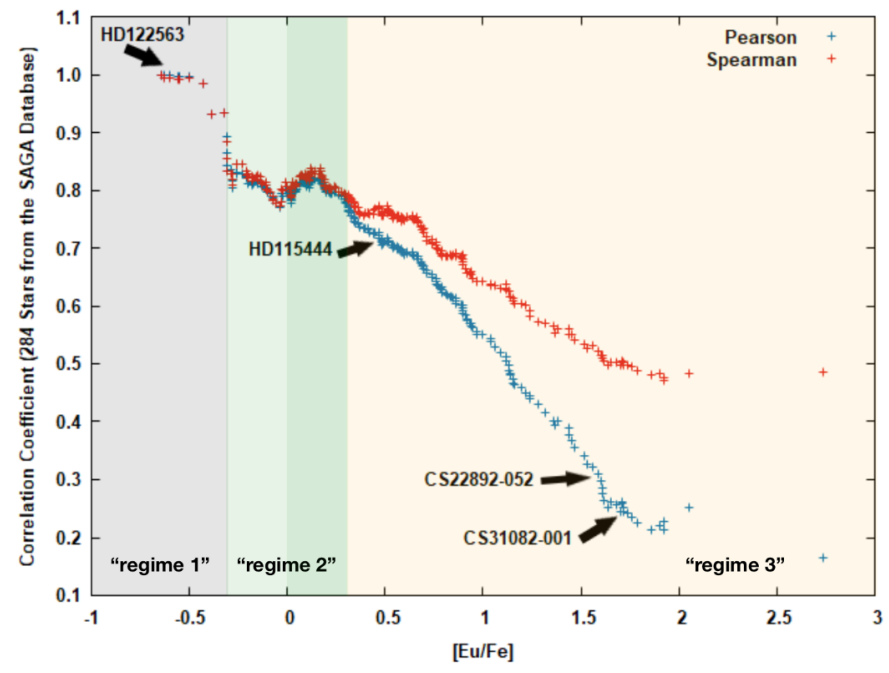

Figure 6. Shown are the Pearson and Spearman correlation coefficients for low-metallicity stars ([Fe/H <-2.5) from the JINAbase database [29], considering stars in an [Eu/Fe] interval with its upper limit displayed on the $\mathrm{x}$-axis. We see for limited-r stars of regime 1 and 2 a high correlation, indicating Fe and Eu co-production, while for r-enriched stars of regime 3 the correlations vanish, arguing for separate nucleosynthesis sources for the Fe and Eu found in these stars. Four typical stars (HD122563, limited-r), HD115444 (r-enriched, r-I), CS22892-052 (r-enriched, r-II), and CS31082-001 (r-enriched, $\mathrm{r}-\mathrm{II}$ with actinide boost) are displayed as well. 
When analyzing data sets of $(\mathrm{x}, \mathrm{y})$ points, the Pearson correlation coefficient measures a possible linear relationship (constant ratio) between their values. In such a case the value of the correlation is either +1 or -1 , depending on whether a linear fit to this relation has a positive or negative slope. The correlation measures also a possible noisiness, the extreme case of a linear fit with a huge $\chi^{2}$, i.e. which does not fit the data at all, stands for a correlation value of 0 . The Spearman correlation coefficient measures correlations in a slightly «milder» way, based on ranks rather than a linear relation. When both methods deviate, this indicates the start of a different correlation pattern [for more information see 30, 31].

When applying this method to correlations between $\mathrm{Eu}$ and $\mathrm{Fe}$ we find the following results (Fig.6) for the Pearson and Spearman correlation coefficients. Among the limited-r stars two types of contributions seem to dominate, we introduced them as categories CI and CII. For both related regimes ( 1 and 2 ) we find a high correlation between $\mathrm{Fe}$ and $\mathrm{Eu}$, indicating the co-production of both elements in the responsible events. In regime 3 (r-enriched stars) a vanishing correlation is strongly supporting sites which produce heavy r-process elements like $\mathrm{Eu}$ uncorrelated (i.e. not co-produced) with Fe. We identify these sites with category CIII events of two types (CIIIa and CIIIb), as indicated from the different strength of r-process production without or with an actinide boost (see Fig.7).

\section{Identifying Astrophysical Sites, their Ejecta Predictions, and Imprint in Low-Metallicity Stars}

If concluding from our statistical analysis that $\mathrm{C} 0$ stars correspond to regular CCSNe, CI and CII stars to quark deconfinement supernovae and magneto-rotational supernovae, that are mainly responsible for the weak r-process in limited-r stars of regime 1 and 2, this suggestion should be tested by comparing the theoretical element predictions for these events with observations of regime 1 and 2 stars. In Table 1 we show this comparison by utilizing the predictions for quark deconfinement (QD) SNe [32] as well as for two sets of magnetorotational (MR) SNe 1 and 2 [33, 34]. QD and MR SNe can reproduce the regime 1 and $2 \mathrm{Sr} / \mathrm{Eu}$ observations well, but in order to reproduce also the observed $\mathrm{Sr} / \mathrm{Fe}$ and $\mathrm{Eu} / \mathrm{Fe}$ values, an additional contribution of $\mathrm{n} \times 0.1 \mathrm{M}_{\odot}$ of Fe with about $\mathrm{n}=200-300$ regular CCSNe is required per QD SN while $n=10$ regular CCSNe per MR SN are needed (comparable with the magnetar fraction of neutron stars). We do not show results from electron-capture supernovae [35], because their $\mathrm{Sr} / \mathrm{Eu}$ ratios are extremely high and their actual existence has been questioned recently [36].

Table 1. Candidates for category CI and CII events

\begin{tabular}{cccccc}
\hline \hline Element ratio & Regime 1 & Regime 2 & MRS1 & MRS2 & QD \\
\hline \hline $\mathrm{Sr} / \mathrm{Eu}$ & $200-750$ & $200-800$ & $438-1300$ & 340 & $142-468$ \\
$\mathrm{Sr} / \mathrm{Fe}$ & $<2 \times 10^{-6}$ & $<2.5 \times 10^{-5}$ & $0.7-5 \times 10^{-2}$ & $2.6 \times 10^{-2}$ & $(5-6) \times 10^{-3}$ \\
$\mathrm{Eu} / \mathrm{Fe}$ & $2 \times 10^{-8}$ & $10^{-7}$ & $1.5-3.6 \times 10^{-5}$ & $7.5 \times 10^{-5}$ & $(1-4) \times 10^{-5}$ \\
$\mathrm{Fe}\left[\mathrm{M}_{\odot}\right]$ & & & $3 \times 10^{-2}$ & $2 \times 10^{-2}$ & $5.5 \times 10^{-2}$ \\
\hline \hline
\end{tabular}

The rank tests in the analysis section have shown that the strong r-process events of category CIII fall into two subcategories without and with an actinide boost. The question is which physical conditions lead to such an actinide boost.

There have been many investigations relating this to fission. Fission cycling affects the Th/Eu ratio and one can find different productions of Eu, $\mathrm{U}$, and Th for different $Y_{e}$ conditions in the r-process. Fig. 7 shows the results of r-process abundance predictions $[38,39]$ as a function 


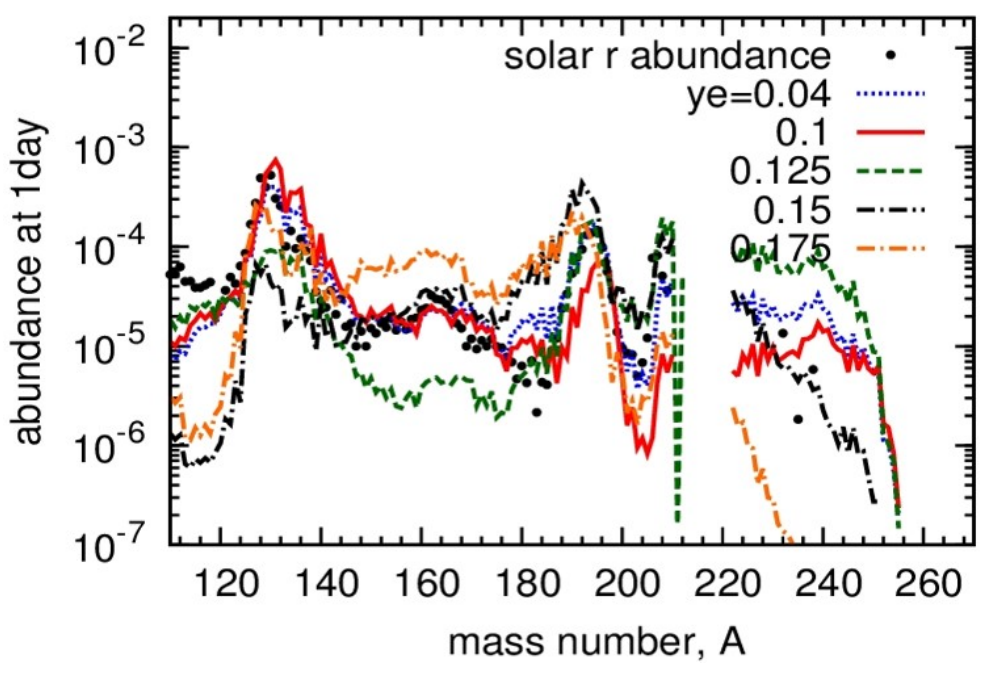

Figure 7. Utilizing the DZ (Duflo-Zucker) mass model [37] one sees large variations of actinide production as a function of $Y_{e}$ in the ejecta. The highest actinide production takes place for a range of 0.125 to 0.15 , decreasing strongly for higher as well as lower $Y_{e}^{\prime} s$ [from 38, 39].

of $Y_{e}$ in the ejecta. In order to explain actinide boosts it requires $Y_{e}$-superpositions in an event which include a sufficient amount of $Y_{e}=0.15$ to 0.125 matter. Results similar to Fig.7 with high Th/Eu values have been obtained in other simulations [40, 41], consistent with $Y_{e}$-values for the ejecta of $\mathrm{BH}$ accretion tori $[42,43]$.

Actinide-boost stars with $\mathrm{Th} / \mathrm{Eu}$ supersolar ( $>0.42$, [44]) exist mostly among r-II stars. This answers the question how we can relate the observed behavior to category CIIIa and CIIIb events by identifying them with NS-mergers and BH accretion tori outflows, e.g. collapsars. This is consistent with the separate rank tests for Th in r-I and r-II stars of Fig.4. Independent of the actinide boost question we test in Table 2 the predicted Eu production for these events (about $10^{-6} \mathrm{M}_{\odot}$ vs. $10^{-4} \mathrm{M}_{\odot}$, when utilizing the results from [43, 45]), combined with about $0.5 \mathrm{M}_{\odot}$ of $\mathrm{Fe}$ for the latter collapsar case. We find that in order to reproduce the observed $\mathrm{Eu} / \mathrm{Fe}$ values in r-I stars (by NS mergers) and r-II stars (by collapsars) requires in both cases the addition of about $\mathrm{n} \times 0.1 \mathrm{M}_{\odot}$ of Fe with $\mathrm{n} \approx 120$, i.e. category CIII events are in the 8 per mil range in relation to $\mathrm{CCSNe}$ [consistent with 46].

Table 2. Candidates for category CIIIa and CIIIb events for complete r-process stars of regime 3

\begin{tabular}{ccccc}
\hline \hline Element ratio & average r-I & average r-II & NS mergers (with Fe from 1 SN) & collapsars \\
\hline \hline $\mathrm{Eu} / \mathrm{Fe}$ & $3 \times 10^{-7}$ & $3 \times 10^{-6}$ & $3.7 \times 10^{-5}$ & $7.4 \times 10^{-5}$ \\
\hline \hline
\end{tabular}

This section followed the initial statistical analysis of the abundance pattern of low-metallicity stars, that came to the conclusion that there exist at least three types of sites for the limited-r, r-I, and r-II stars. We actually found a possible division among limited-r stars and suggested four categories: I for QD supernovae (a hint from Fig.6 in regime 1, although not clearly proven), II for magneto-rotational supernovae for explaining regime 2, IIIa for neutron star mergers, and IIIb for collapsars/hypernovae for explaining regime 3. For those four categories there exist abundance patterns (and ejecta mass) predictions from theoretical models. Utilizing these predictions we asked the question how the observed $\mathrm{Sr} / \mathrm{Eu}, \mathrm{Sr} / \mathrm{Fe}$, and $\mathrm{Eu} / \mathrm{Fe}$ could 
be reproduced among limited-r, r-I, and r-II stars. The result was that (with a slightly unclear option for QD-supernovae) limited-r stars could be reproduced when magneto-rotational supernovae would occur with a frequency of $1 / 10(n=10$ in Table 1$)$ of regular supernovae (which is consistent with the fraction of magnetars among neutron stars), and r-I and r-II stars could be reproduced with neutron star merger and collapsars if the occur with a frequence of a few permille of core-collapse supernovae $(n \approx 120$ in Table 2$)$. For neutron star mergers this is consistent with the frequency based on LIGO and kilonova observations. For collapsars this makes sense but the models are not sufficiently advanced, yet, in order for a full proof.

\section{Summary}

Is the large scatter in r-process abundances with respect to those of other elements (e.g. Eu vs. $\mathrm{Fe}$ ) at low metallicities only the result of inhomogeneous admixture from rare events, or do the also observed variations in r-process abundance patterns point to different origins? Such questions have been addressed in many galactic chemical evolution studies [e.g. 8, 9, $11,12]$. There exist attempts to explain the variation in abundance patterns all by neutron star merger events with varying neutron star masses and also mass symmetries/asymmetries in the binary systems [47], including systems with fast $\mathrm{BH}$ formation which are part of our category CIIIb. With the correlation / no correlation tests of e.g. Eu with Fe, we suggest different sources resulting either in a weak r-process with Fe co-production or a strong r-process with no or negligible Fe co-production. The effect that the r-process (or Eu) production in these events (magneto-rotational supernovae, NS mergers, collapsars) also increases among these categories by about a factor of 10 for each, permits an explanation of the limited-r, r-I, and r-II patterns, combined with the inhomogeneous evolution in the early Galaxy.

We can formulate a consistent picture, utilizing existing abundance pattern predictions for these events, related to r-process ejecta masses and event frequencies, which are in accordance with category CI and CII events being less frequent than regular core-collapse supernovae by about a factor of 10 , and category CIIIa and CIIIb events being less frequent by a factor of 100 to 1000 . This seems consistent with the first Fe from core-collapse supernovae showing up at metallicities as low as $[\mathrm{Fe} / \mathrm{H}] \lessgtr-5$, the first input from category CI and CII events at about $[\mathrm{Fe} / \mathrm{H}] \approx-4$, and the first input from category CIIIa and $\mathrm{CIII}$ events at about $[\mathrm{Fe} / \mathrm{H}] \approx-3$. This implies that stars with metallicities less than $[\mathrm{Fe} / \mathrm{H}] \approx-2.5$ do not necessarily show the imprint of only one nucleosynthesis event, but possibly a superposition. This indicates that most of the Fe is going back to frequent core-collapse supernovae, opposite to the majority of r-process elements, originating from a variety of rarer events. These conclusions are still somewhat speculative, they should be tested against further observational and modeling progress. More details of our present study can be found in ref. [48].

Acknowledgement. The investigations of this paper would not have been possible without the publicly available observational SAGA and JINA data bases [16, 29], and we want to express our thanks to the authors of these tremendous research resources. Furthermore, the COST actions ChETEC (Chemical Elements as Tracers of the Evolution of the Cosmos, CA16117), GWvese (Gravitational waves, black holes and fundamental physics, CA16104), and Pharos (The multi-messenger physics and astrophysics of neutron stars, CA16214) provided an inspiring atmosphere for thoughts along the lines discussed here. SR has been supported by the Swedish Research Council (VR) under grant numbers 2016-03657_3 and 2020-05044, by the Swedish National Space Board under grant number Dnr. 107/16, the research environment grant "Gravitational Radiation and Electromagnetic Astrophysical Transients (GREAT)" funded by the Swedish Research council (VR) under Dnr 2016-06012 and by the Knut and Alice Wallenberg Foundation (KAW 2019.0112). 


\section{References}

[1] J.M. Lattimer, D.N. Schramm, Astrophys. J. 192, L145 (1974)

[2] R.D. Hoffman, S.E. Woosley, Y.Z. Qian, Astrophys. J. 482, 951 (1997)

[3] C. Freiburghaus, J.F. Rembges, T. Rauscher, E. Kolbe, F.K. Thielemann, K.L. Kratz, B. Pfeiffer, J.J. Cowan, Astrophys. J. 516, 381 (1999)

[4] C. Freiburghaus, S. Rosswog, F.K. Thielemann, Astrophys. J. 525, L121 (1999)

[5] K. Farouqi, K. Kratz, B. Pfeiffer, T. Rauscher, F. Thielemann, J.W. Truran, Astrophys. J. 712, 1359 (2010)

[6] J.J. Cowan, C. Sneden, J.E. Lawler, A. Aprahamian, M. Wiescher, K. Langanke, G. Martinez-Pinedo, F.K. Thielemann, Rev. Mod. Phys. 93, 015002 (2021)

[7] F. Matteucci, D. Romano, A. Arcones, O. Korobkin, S. Rosswog, Mon. Not. Roy. Astron. Soc. 438, 2177 (2014)

[8] G. Cescutti, D. Romano, F. Matteucci, C. Chiappini, R. Hirschi, Astron. \& Astrophys. 577, A139 (2015)

[9] B. Wehmeyer, M. Pignatari, F.K. Thielemann, Mon. Not. Roy. Astron. Soc. 452, 1970 (2015)

[10] S.G. Ryan, J.E. Norris, T.C. Beers, Astrophys. J. 471, 254 (1996)

[11] C. Kobayashi, A.I. Karakas, M. Lugaro, Astrophys. J. 900, 179 (2020)

[12] F. van de Voort, R. Pakmor, R.J.J. Grand, V. Springel, F.A. Gómez, F. Marinacci, Mon. Not. Roy. Astron. Soc. 494, 4867 (2020)

[13] D.L. Burris, C.A. Pilachowski, T.E. Armandroff, C. Sneden, J.J. Cowan, H. Roe, Astrophys. J. 544, 302 (2000)

[14] R. Cayrel, V. Hill, T.C. Beers, B. Barbuy, M. Spite, F. Spite, B. Plez, J. Andersen, P. Bonifacio, P. François et al., Nature 409, 691 (2001)

[15] S. Honda, W. Aoki, Y. Ishimaru, S. Wanajo, S.G. Ryan, Astrophys. J. 643, 1180 (2006)

[16] T. Suda, Y. Katsuta, S. Yamada, T. Suwa, C. Ishizuka, Y. Komiya, K. Sorai, M. Aikawa, M.Y. Fujimoto, Publ. Astron. Soc. Japan 60, 1159 (2008)

[17] H. Schatz, R. Toenjes, B. Pfeiffer, T.C. Beers, J.J. Cowan, V. Hill, K.L. Kratz, Astrophys. J. 579, 626 (2002)

[18] I.U. Roederer, K.L. Kratz, A. Frebel, N. Christlieb, B. Pfeiffer, J.J. Cowan, C. Sneden, Astrophys. J. 698, 1963 (2009)

[19] I.U. Roederer, C. Sneden, J.E. Lawler, J.J. Cowan, Astrophys. J. 714, L123 (2010)

[20] L. Mashonkina, N. Christlieb, K. Eriksson, Astron. \& Astrophys. 569, A43 (2014)

[21] I.U. Roederer, Astrophys. J. 835, 23 (2017)

[22] E.M. Holmbeck, T.C. Beers, I.U. Roederer, V.M. Placco, T.T. Hansen, C.M. Sakari, C. Sneden, C. Liu, Y.S. Lee, J.J. Cowan et al., Astrophys. J. 859, L24 (2018)

[23] T.T. Hansen, E.M. Holmbeck, T.C. Beers, V.M. Placco, I.U. Roederer, A. Frebel, C.M. Sakari, J.D. Simon, I.B. Thompson, Astrophys. J. 858, 92 (2018)

[24] C.M. Sakari et al., Astrophys. J. 868, 110 (2018)

[25] E.M. Holmbeck, T.T. Hansen, T.C. Beers, V.M. Placco, D.D. Whitten, K.C. Rasmussen, I.U. Roederer, R. Ezzeddine, C.M. Sakari, A. Frebel et al., Astrophys. J. Suppl. 249, 30 (2020)

[26] R. Ezzeddine, K. Rasmussen, A. Frebel, A. Chiti, K. Hinojisa, V.M. Placco, A.P. Ji, T.C. Beers, T.T. Hansen, I.U. Roederer et al., Astrophys. J. 898, 150 (2020)

[27] D. Yong, C. Kobayashi, G.S. Da Costa, M.S. Bessell, A. Chiti, A. Frebel, K. Lind, A.D. Mackey, T. Nordlander, M. Asplund et al., Nature 595, 223 (2021), 2107.03010 
[28] B.S. Everitt, S. Landau, M. Leese, D. Stahl, Cluster Analysis, 5th Edition, Wiley Series in Probability and Statistics (Wiley, New York, 2011), ISBN 978-0-470-97844-3

[29] A. Abohalima, A. Frebel, Astrophys. J. Suppl. 238, 36 (2018)

[30] A. Tamhane, D. Dunlop, Statistics and Data Analysis (Prentice-Hall, Upper Saddle River, 2000)

[31] D. Spiegelhalter, The art of statistics (Penguin Random House, Milton Keynes, 2019)

[32] T. Fischer, M.R. Wu, B. Wehmeyer, N.U.F. Bastian, G. Martínez-Pinedo, F.K. Thielemann, Astrophys. J. 894, 9 (2020)

[33] N. Nishimura, H. Sawai, T. Takiwaki, S. Yamada, F.K. Thielemann, Astrophys. J. 836, L21 (2017)

[34] M. Reichert, M. Obergaulinger, M. Eichler, M.Á. Aloy, A. Arcones, Mon. Not. Roy. Astron. Soc. 501, 5733 (2021)

[35] S. Wanajo, H.T. Janka, B. Müller, Astrophys. J. 726, L15 (2011)

[36] O.S. Kirsebom et al., Phys. Rev. Lett. 123, 262701 (2019)

[37] J. Duflo, A.P. Zuker, Phys. Rev. C 52, R23 (1995)

[38] F.K. Thielemann, B. Wehmeyer, M.R. Wu, r-Process Sites, their Ejecta Composition, and their Imprint in Galactic Chemical Evolution, in Journal of Physics Conference Series (2020), Vol. 1668 of Journal of Physics Conference Series, p. 012044

[39] M.R. Wu et al. (2017), unpublished

[40] E.M. Holmbeck, T.M. Sprouse, M.R. Mumpower, N. Vassh, R. Surman, T.C. Beers, T. Kawano, Astrophys. J. 870, 23 (2019)

[41] M. Eichler, W. Sayar, A. Arcones, T. Rauscher, Astrophys. J. 879, 47 (2019)

[42] R. Fernández, A. Tchekhovskoy, E. Quataert, F. Foucart, D. Kasen, Mon. Not. Roy. Astron. Soc. 482, 3373 (2019)

[43] D.M. Siegel, J. Barnes, B.D. Metzger, Nature 569, 241 (2019)

[44] K. Lodders, Space Sci. Rev. 217, 44 (2021)

[45] B. Côté, K. Belczynski, C.L. Fryer, C. Ritter, A. Paul, B. Wehmeyer, B.W. O'Shea, Astrophys. J. 836, 230 (2017)

[46] S. Rosswog, U. Feindt, O. Korobkin, M.R. Wu, J. Sollerman, A. Goobar, G. MartinezPinedo, Class. Quantum Gravity 34, 104001 (2017)

[47] E.M. Holmbeck, A. Frebel, G.C. McLaughlin, R. Surman, R. Fernández, B.D. Metzger, M.R. Mumpower, T.M. Sprouse, Astrophys. J. 909, 21 (2021)

[48] K. Farouqi, F.K. Thielemann, S. Rosswog, K.L. Kratz, arXiv e-prints arXiv:2107.03486 (2021) 